\title{
Geant4-DNA simulation of the pre-chemical stage of water radiolysis and its impact on initial radiochemical yields
}

\author{
Wook-Geun Shin ${ }^{1,2,3}$, Jose Ramos-Mendez ${ }^{4}$, Ngoc Hoang $\operatorname{Tran}^{1}$, Shogo Okada ${ }^{5}$, \\ Yann Perrot ${ }^{6}$, Carmen Villagrasa ${ }^{6}$, and Sebastien Incerti ${ }^{1}$ \\ ${ }^{1} U M R$ 5797, Bordeaux University, CNRS, CENBG, 33170 Gradignan, France \\ ${ }^{2}$ Department of Radiation Oncology, Seoul National University Hospital, 03080 Seoul, Korea \\ ${ }^{3}$ Biomedical Research Institute, Seoul National University Hospital, 03080 Seoul, Korea \\ ${ }^{4}$ Department of Radiation Oncology, University of California San Francisco, San Francisco, 94143 CA, USA \\ ${ }^{5}$ KEK, 1-1, Oho, Tsukuba, Ibaraki 305-0801, Japan \\ ${ }^{6}$ IRSN, Institut de Radioprotection et de Sûreté Nucléaire, BP17, 92262 Fontenay-aux-Roses, France
}

\begin{abstract}
This paper demonstrates the impact of the pre-chemical stage, especially the dissociation scheme and the associated probabilities, on water radiolysis simulation using the Geant4-DNA Monte Carlo track structure simulation toolkit. The models and parameters provided by TRACs have been collected and implemented into Geant4-DNA. In order to evaluate their influence on water radiolysis simulation, the radiochemical yields (G-values) are evaluated as a function of time and LET using the "chem6" Geant4-DNA example, and they are compared with published experimental and calculated data. The new pre-chemical models lead to a better agreement with literature data than the default pre-chemical models of Geant4-DNA, especially for $\cdot \mathrm{OH}$ radicals and $\mathrm{H} 2 \mathrm{O} 2$. The revised chemistry constructor "G4EmDNAChemistry_option3" is available in Geant4-DNA version 10.7.
\end{abstract}

\section{Introduction}

The mechanistic simulation of biological effects induced by ionizing radiation at the subcellular and DNA scales is a scientific challenge. Such an approach could lead to a better understanding of carcinogenesis at low doses and to the development of more accurate risk models [1]. Numerous Monte Carlo track structure (MCTS) codes have been developed so far to predict DNA damage induction. Among them, Geant4-DNA [2-5], PARTRAC [6], TRACs (TRACELE and TRACPRO) [7], and others (see comprehensive reviews in [8-10]), can predict both direct and 
indirect damage, from physical interactions and water radiolysis products, respectively. At the subcellular scale, it is reported that the contribution of indirect damages becomes more important than that of direct damage below a linear energy transfer (LET) of $300 \mathrm{keV} / \mu \mathrm{m}$. [11]. Therefore, a careful modeling of water radiolysis in the cellular medium (liquid water in these codes) is essential.

The "pre-chemical" stage of water radiolysis links the "physical" and "chemical" stages and determines the initial radiochemical yields, called G-values, which are the number of molecular species normalized per $100 \mathrm{eV}$ of deposited energy. During the pre-chemical stage, the excited and ionized water molecules, $\mathrm{H}_{2} \mathrm{O}^{*}$ and $\mathrm{H}_{2} \mathrm{O}^{+}$, are dissociated into molecular species according to the pre-chemical model. To date, it is unfortunately almost impossible to experimentally probe the prechemical stage on the femtosecond scale. Therefore, it is still common in radiochemical studies to adjust the pre-chemical parameters to match the simulated radiochemical yields to the experimental data, due to the lack of a complete mechanistic understanding of the pre-chemical processes. For example, PARTRAC uses the pre-chemical model of TRACs with modifications of the dissociation scheme and probabilities to reproduce the experimental yields of chemical species. On the other hand, the pre-chemical model of Geant4-DNA (version 10.7) is derived from PARTRAC without any adjustment, but additional physico-chemical processes such as electron attachment and electron-hole recombination are added. These additional processes, indeed more realistic, induce a significant disagreement which makes it difficult to match the experimental data for radiolysis of water.

Thus, in this study, we propose to revise the pre-chemical dissociation scheme and probabilities of the 10.7 version of Geant4-DNA to improve the agreement with the experimental and simulated G-values found in the literature, as a function of time and LET. 


\section{Materials and methods}

\subsection{Pre-chemical models}

Table 1. Dissociation scheme and associated probability for each channel used in this work, and available in Geant4-DNA, PARTRAC and TRACs MCTS codes. Values in italic indicate changes compared to Geant4-DNA 10.7 [12].

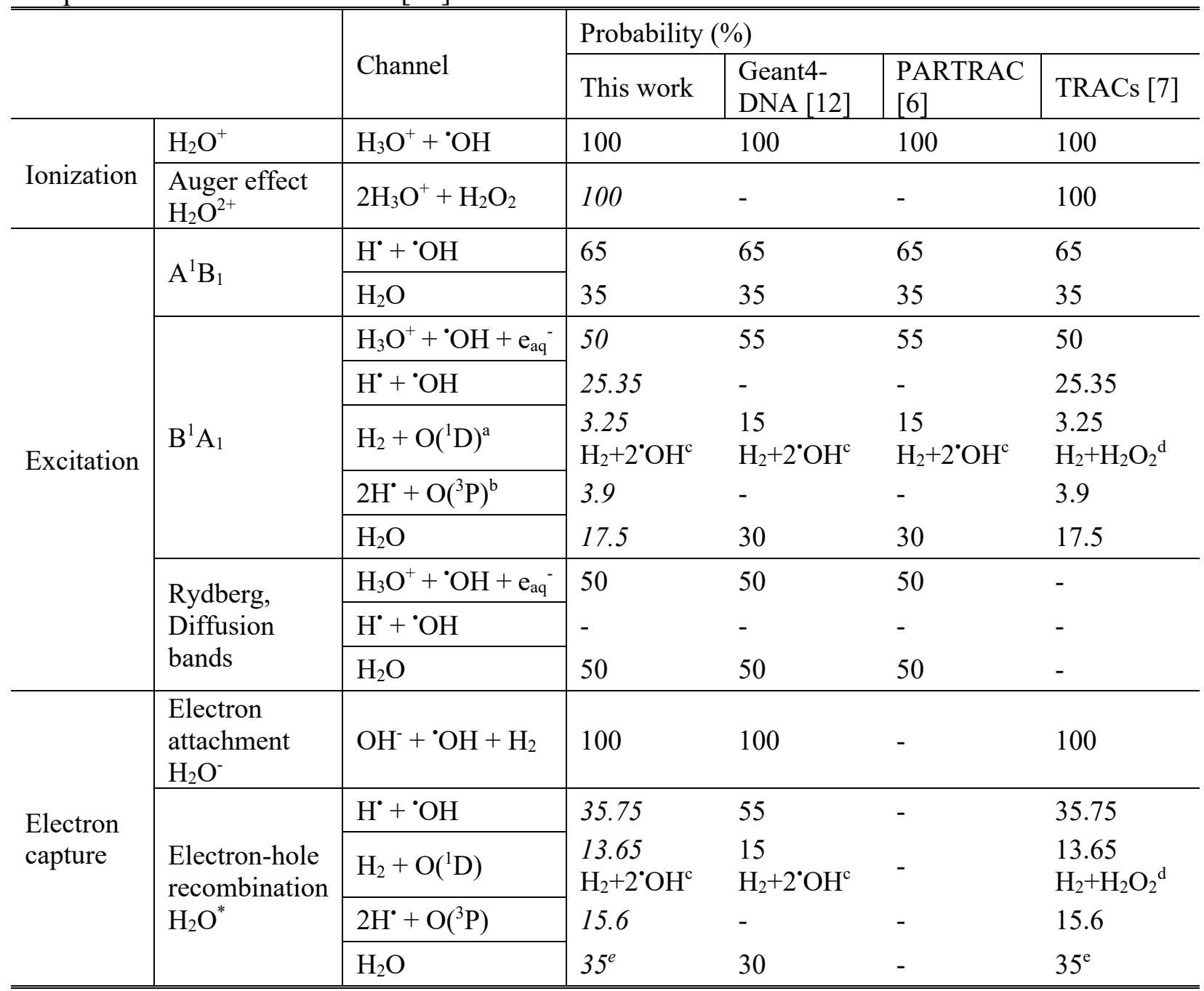

${ }^{a}$ Oxygen atom in the singlet $\mathrm{D}$ state.

${ }^{\mathrm{b}}$ Oxygen atom in the triplet $\mathrm{P}$ state.

${ }^{\mathrm{c}} \mathrm{O}\left({ }^{1} \mathrm{D}\right)+\mathrm{H}_{2} \mathrm{O} \rightarrow 2^{\circ} \mathrm{OH}$ from Burns et al., 1981 [13].

${ }^{d} \mathrm{O}\left({ }^{1} \mathrm{D}\right)+\mathrm{H}_{2} \mathrm{O} \rightarrow \mathrm{H}_{2} \mathrm{O}_{2}$ from Taube, 1956 [14].

e Same relaxing probability for $\mathrm{B}^{1} \mathrm{~A}_{1}$ excitation except auto-ionization.

The dissociation scheme of ionized water molecules $\left(\mathrm{H}_{2} \mathrm{O}^{+}\right)$is clearly established [15], ${ }^{\circ} \mathrm{OH}$ and $\mathrm{H}_{3} \mathrm{O}^{+}$, regardless of the ionization level. Furthermore, in this work, the dissociation of $\mathrm{H}_{2} \mathrm{O}^{2+}$ 
induced by the Auger process is newly implemented in Geant4-DNA. The excited water molecule $\left(\mathrm{H}_{2} \mathrm{O}^{*}\right)$ is dissociated according to the five excited states. The $\mathrm{A}^{1} \mathrm{~B}_{1}$ state represents the excitation of the $5^{\text {th }}$ electronic layer $\left(1 \mathrm{~b}_{1} \rightarrow 4 \mathrm{a}_{1}\right)$, while the $\mathrm{B}^{1} \mathrm{~A}_{1}$ state corresponds to the excitation of the $4^{\text {th }}$ layer $\left(3 a_{1} \rightarrow 4 a_{1}\right)$, and the other states represent Rydberg series and diffusion bands.

As shown in Table 1, Geant4-DNA uses the pre-chemical model of PARTRAC to describe the dissociation scheme after excitation, and considers two additional processes (after electron capture) that are not considered by PARTRAC: electron attachment and electron-hole recombination. Thus, in this study, we applied the $\mathrm{B}^{1} \mathrm{~A}_{1}$ dissociation channel proposed by TRACs [7]. Note that the different atomic oxygen states $\left({ }^{1} \mathrm{D}\right.$ or $\left.{ }^{3} \mathrm{P}\right)$ are considered as different species in the water radiolysis simulation, and we decided to use the more recent dissociation channel of $O\left({ }^{1} \mathrm{D}\right)$ into two hydroxyl radicals proposed by Burns et al. [13] instead of that of Taube [14] (which considers dissociation into $\mathrm{H}_{2} \mathrm{O}_{2}$ ) in order to obtain better agreement with experimental data.

The dissociation of $\mathrm{H}_{2} \mathrm{O}^{*}$ after electron-hole recombination is accounted for in both TRACs and Geant4-DNA. For this process, an empirical dissociation channel was proposed by Rowe et al. [16], in which it is reported that the fraction of the $\mathrm{H}^{\cdot}+{ }^{\cdot} \mathrm{OH}$ dissociation channel is $55 \%$ of the species produced. However, the 10.7 version of Geant4-DNA directly uses this value without reducing it by considering the relaxation probability. Therefore, we corrected the dissociation probabilities of electron-hole recombination taking into account those of relaxation probability, for example $35.75 \%$ for $\mathrm{H}^{\cdot}+{ }^{\circ} \mathrm{OH}$ channel $(55 \% \times 65 \%)$ [7], for consistency with the original paper by Rowe et al. [16].

In summary, this study includes Auger-generated $\mathrm{H}_{2} \mathrm{O}^{2+}$ dissociation, dissociation channel modifications for $\mathrm{B}^{1} \mathrm{~A}_{1}$ excitation, and the electron-hole recombination process.

\subsection{Simulation configuration}


The public version of Geant4-DNA (version 10.7) and the recently implemented independent reaction times (IRT) technique (available in the example "chem6") $[17,18]$ are used in this study to simulate G-values for incident electrons, protons and alpha particles. The simulation configurations and physics settings validated in our previous water radiolysis study [19-22] are used, namely the G4EmDNAPhysics_option2 physics constructor with the ELSEPA electron elastic scattering model and the Meesungnoen electron thermalization model. For radiolysis simulation, we choose the G4EmDNAChemistry_option3 chemistry constructor, which uses the same chemistry parameters as RITRACKS from Elliot's work [23, 24], and the proposed modification of the pre-chemical parameters presented in this work.

Computational and experimental data sets presented by [25-39] are used for comparison with the simulated G-values as a function of time. For G-values as a function of LET, computational and experimental data obtained by [22, 40-45] are compared with the results. It should be noted that most of the experimental data are measured under arbitrary control of the solvent concentration, whereas the simulations are performed for pure liquid water.

\section{Results and discussion}

\subsection{G-values as a function of time}



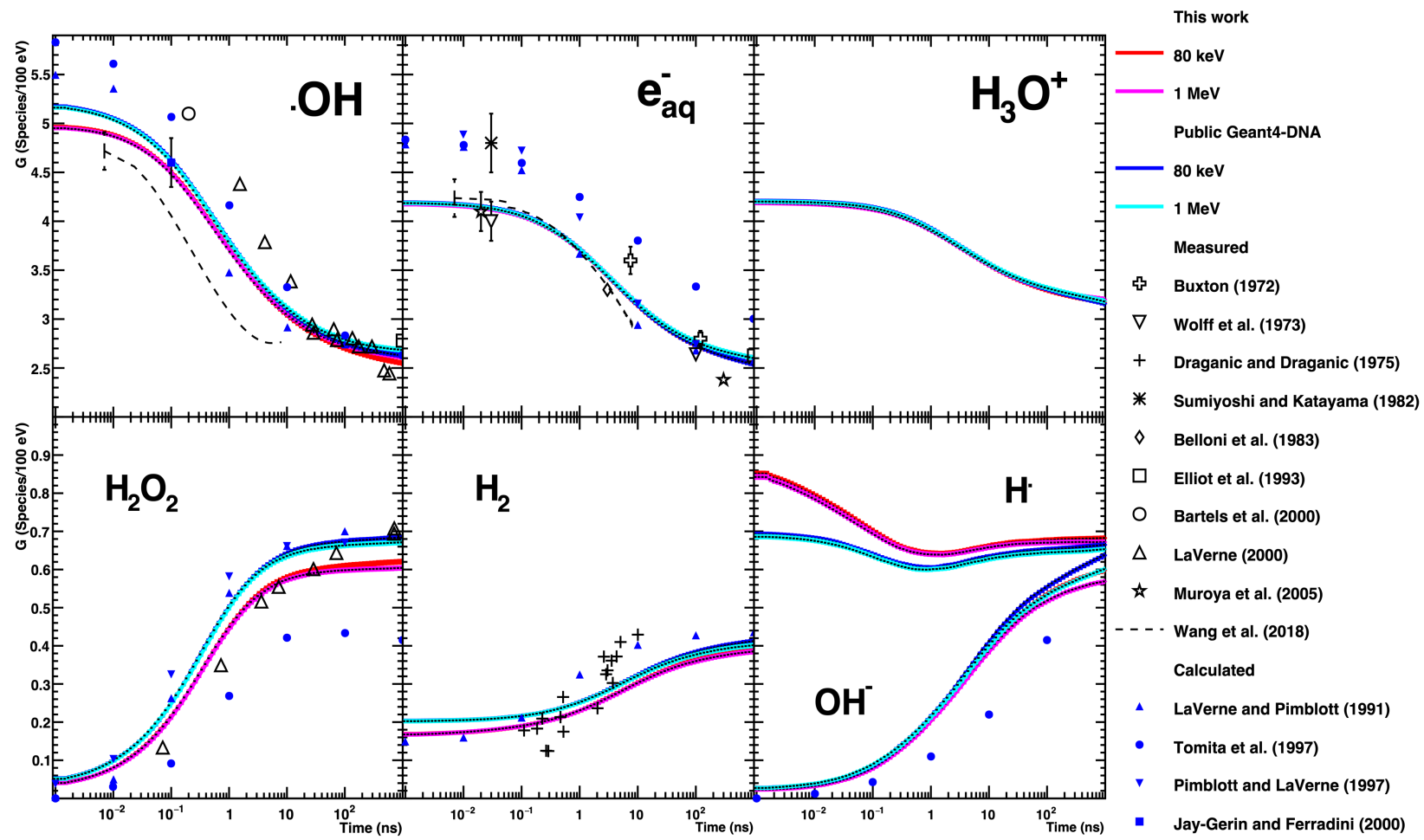

Figure 1. The time evolution of G-values for incident electrons calculated with the modifications proposed in this work (red and magenta curves) and by the 10.7 version of Geant4-DNA (blue and cyan curves), compared to the measured and calculated yields. For Wang et al. (2018), the fit data are shown.

We first present in Figure 1 the simulated radiochemical yields as a function of time, using Geant4-DNA 10.7 (blue and cyan curves) and the improved pre-chemical stage proposed in this work (red and magenta curves). The LET values of all calculated and measured references are between $0.186-0.314 \mathrm{keV} / \mu \mathrm{m}$. Thus, only the results for $80 \mathrm{keV}$ and $1 \mathrm{MeV}$ electrons, whose LET values are 0.61 and $0.16 \mathrm{keV} / \mu \mathrm{m}$, respectively, are shown in Figure 1.

The G-values are not much affected by the LET changes in the low LET region, for example, between the 0.16 and $0.61 \mathrm{keV} / \mu \mathrm{m}$ curves. Thus, we can conclude that the variation observed in the experimental data comes mainly from the different experimental setups. The red and magenta curves have lower G-values for ${ }^{\circ} \mathrm{OH}$ radicals, $\mathrm{H}_{2} \mathrm{O}_{2}, \mathrm{H}_{2}$, and $\mathrm{OH}^{-}$than the blue and cyan curves. The G-values of these species calculated in this study are closer to recent experimental data such 
as those of Wang et al. [39] (dashed line, for ${ }^{\circ} \mathrm{OH}$ and $\mathrm{e}_{\mathrm{aq}}{ }^{-}$), measured at the shortest time after irradiation (7 ps), within the experimental uncertainty.

For $\mathrm{H}_{2}$ molecules, the initial G-value with the new pre-chemical parameters is 0.16 molecules/100 eV at $1 \mathrm{ps}$. This value is closer to the experimental estimate of Schwarz et al. [46] reported at 0.15 molecules/100 eV (shown as the initial value of LaVerne and Pimblott [30]).

We observed that the differences between both values on ${ }^{\circ} \mathrm{OH}$ and $\mathrm{H}^{\circ}$ radicals saturate at a late time. However, up to $5 \mathrm{~ns}$ (which is the proposed time limit of the damage simulation in Geant4DNA [47]), the G-values of 'OH radical (which are mainly responsible for indirect DNA damage) decrease and the two models differ by up to $5 \%$. 


\subsection{G-values as a function of LET}

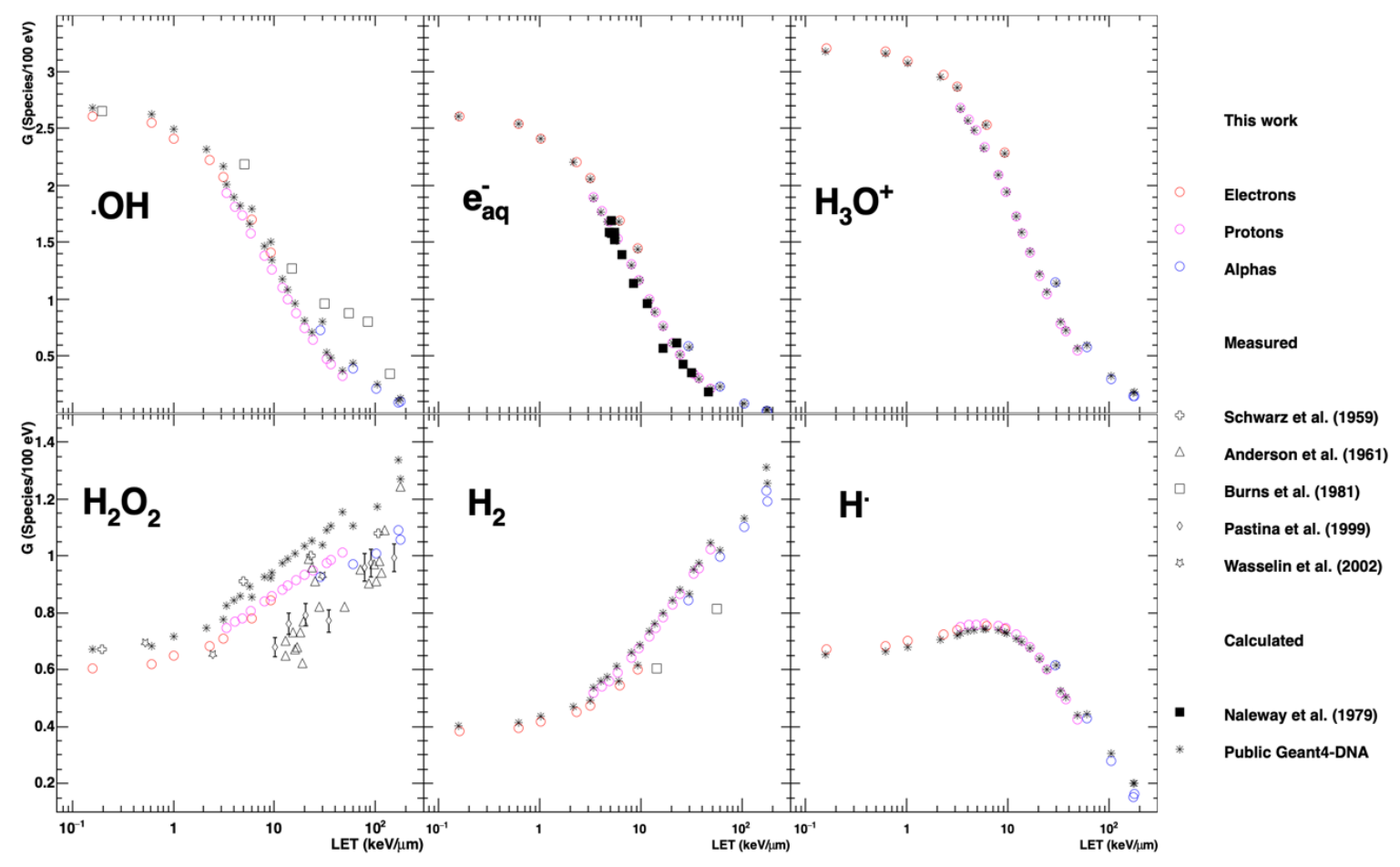

Figure 2. The G-values at the end of the chemical stage ( $1 \mu \mathrm{s})$ as a function of LET for incident electrons, protons and alphas (open coloured circles), compared with measured and calculated yields.

Next, G-values as a function of LET in the range of $0.16-177.85 \mathrm{keV} / \mu \mathrm{m}$ are calculated at 1 $\mu$ s, as shown in Figure 2. We can observe a slight change for ${ }^{\circ} \mathrm{OH}, \mathrm{H}_{2}$, and $\mathrm{H}^{\bullet}$ due to the correction of dissociation probabilities in the recombination process and $\mathrm{B}^{1} \mathrm{~A}_{1}$ excitation. The main improvement here is that the G-values of $\mathrm{H}_{2} \mathrm{O}_{2}$, which previously showed higher values than literature data using the 10.7 version of Geant4-DNA, are smaller due to the decrease in the initial number of ${ }^{\circ} \mathrm{OH}$ radicals at the beginning of the chemical stage.

\section{Conclusion}

In this study, the pre-chemical stage of Geant4-DNA is evaluated by comparing it with the 
TRACs MCTS codes. The Geant4-DNA dissociation scheme is then revised to improve agreement with experimental and computational G-values as a function of time and LET found in the literature.

The new dissociation scheme including $\mathrm{B}^{1} \mathrm{~A}_{1}$ excitation and the electron-hole recombination process directly influences the G-values of ${ }^{\circ} \mathrm{OH}, \mathrm{H}_{2}$, and $\mathrm{H}^{\bullet}$ species. Our results are close to recent experimental data, especially for ${ }^{\circ} \mathrm{OH}$ and $\mathrm{H}_{2} \mathrm{O}_{2}$ generated by ${ }^{\circ} \mathrm{OH}+{ }^{\circ} \mathrm{OH}$ reactions. This adjustment could reduce the Geant4-DNA simulated indirect DNA damage yields, which were relatively higher than experimental data [18]; this will be the subject of another study. The new pre-chemical model presented in this work will be soon released in Geant4-DNA as an alternative option.

In future work, the dissociation of $\mathrm{H}_{2} \mathrm{O}^{+}$cation [48] will be improved and multi-ionized water molecules [49] will be added based on recent literature for the accurate modeling of pre-chemical stage.

\section{References}

[1] Mullenders L, Atkinson M, Paretzke H, Sabatier L, Bouffler S. Assessing cancer risks of low-dose radiation. Nat Rev Cancer. 2009;9:596-604.

[2] Bernal MA, Bordage MC, Brown JMC, Davidkova M, Delage E, El Bitar Z, et al. Track structure modeling in liquid water: A review of the Geant4-DNA very low energy extension of the Geant4 Monte Carlo simulation toolkit. Phys Med. 2015;31:861-74.

[3] Incerti S, Ivanchenko A, Karamitros M, Mantero A, Moretto P, Tran H, et al. Comparison of GEANT4 very low energy cross section models with experimental data in water. Med Phys. 2010;37:4692-708.

[4] Incerti S, Baldacchino G, Bernal M, Capra R, Champion C, Francis Z, et al. The Geant4-DNA project. Int J Model Simul Sci Comput. 2010;1:157-78.

[5] Incerti S, Kyriakou I, Bernal M, Bordage MC, Francis Z, Guatelli S, et al. Geant4-DNA example applications for track structure simulations in liquid water: a report from the Geant4-DNA Project. Med Phys. 2018;45:e722-e39.

[6] Kreipl MS, Friedland W, Paretzke HG. Time- and space-resolved Monte Carlo study of water radiolysis for photon, electron and ion irradiation. Radiat Environ Bioph. 2009;48:11-20.

[7] Cobut V, Frongillo Y, Patau JP, Goulet T, Fraser M-J, Jay-Gerin JP. Monte Carlo simulation of fast electron and proton tracks in liquid water-I. Physical and physicochemical aspects. Radiat Phys Chem. 1998;51:229-43.

[8] Nikjoo H, Uehara S, Emfietzoglou D, Cucinotta FA. Track-structure codes in radiation research. Radiat Meas. 2006;41:1052-74.

[9] El Naqa I, Pater P, Seuntjens J. Monte Carlo role in radiobiological modelling of radiotherapy outcomes. Phys Med Biol. 2012;57:R75-97.

[10] Nikjoo H, Emfietzoglou D, Liamsuwan T, Taleei R, Liljequist D, Uehara S. Radiation track, DNA damage and response-a review. Rep Prog Phys. 2016;79:116601.

[11] Hirayama R, Ito A, Tomita M, Tsukada T, Yatagai F, Noguchi M, et al. Contributions of direct and indirect actions in cell killing by high-LET radiations. Radiat Res. 2009;171:212-8.

[12] Karamitros M, Mantero A, Incerti S, Friedland W, Baldacchino G, Barberet P, et al. Modeling radiation chemistry in the Geant4 toolkit. Prog Nucl Sci Tech. 2011;2:503-8.

[13] Burns WG, Marsh WR. Radiation chemistry of high-temperature (300-410 deg) water. J Chem Soc Faraday 
Trans 1. 1981;77:197-215.

[14] Taube H. Photochemical reactions of ozone in solution. Trans Faraday Soc. 1956;53:656-65.

[15] Thomsen CL, Madsen D, Keiding SR, Thøgersen J, Christiansen O. Two-photon dissociation and ionization of liquid water studied by femtosecond transient absorption spectroscopy. J Chem Phys. 1999;110:3453-62.

[16] Rowe BR, Vallée F, Queffelec JL, Gomet JC, Morlais M. The yield of oxygen and hydrogen atoms through dissociative recombination of H2O+ ions with electrons. J Chem Phys. 1988;88:845-50.

[17] Ramos-Mendez J, Shin WG, Dominguez-Kondo JN, Incerti S, Tran HN, Villagrasa C, et al. Independent reaction times method in Geant4-DNA: implementation and performance. Med Phys. 2020;47:5919-30.

[18] Shin WG. Development and application of the Geant4-DNA toolkit for the simulation of radiobiological effects at the sub-cellular scale. Bordeaux: Bordeaux and Yonsei University; 2021.

[19] Shin WG, Bordage MC, Emfietzoglou D, Kyriakou I, Sakata D, Min CH, et al. Development of a new Geant4DNA electron elastic scattering model for liquid-phase water using the ELSEPA code. J Appl Phys. 2018;124.

[20] Shin WG, Ramos-Mendez J, Faddegon B, Tran HN, Villagrasa C, Perrot Y, et al. Evaluation of the influence of physical and chemical parameters on water radiolysis simulations under $\mathrm{MeV}$ electron irradiation using Geant4-

DNA. J Appl Phys. 2019;126.

[21] Karamitros M, Luan S, Bernal MA, Allison J, Baldacchino G, Davidkova M, et al. Diffusion-controlled reactions modeling in Geant4-DNA. J Comput Phys. 2014;274:841-82.

[22] Ramos-Mendez J, Perl J, Schuemann J, McNamara A, Paganetti H, Faddegon B. Monte Carlo simulation of chemistry following radiolysis with TOPAS-nBio. Phys Med Biol. 2018;63:105014.

[23] Elliot AJ. Rate constants and G-values for the simulation of the radiolysis of light water over the range 0-300 deg. Ontario, Canada: Chalk River Laboratories; 1994.

[24] Plante I, Devroye L. Considerations for the independent reaction times and step-by-step methods for radiation chemistry simulations. Radiat Phys Chem. 2017;139:157-72.

[25] Buxton GV. Nanosecond pulse radiolysis of aqueous solution containing oproton and hydroxyl radical scavengers. Proc R Soc A. 1972;328:9-21.

[26] Wolff RK, Bronskill MJ, Aldrich JE, Hunt JW. Picosecond pulse radiolysis. IV. Yield of the solvated electron at 30 picoseconds. J Phys Chem. 1973;77:1350-5.

[27] Draganić ZD, Draganić IG. Formation of primary reducing yields (Geaq- and GH2) in the radiolysis of aqueous solutions of some positive ions. Int J Radiat Phys Chem. 1975;7:381-6.

[28] Burns WG, May R, Buxton GV, Wilkinson-Tough GS. Nanosecond proton pulse radiolysis of aqueous solutions. J Chem Soc Faraday Trans. 1981;77:1543-51.

[29] Sumiyoshi T, Katayama M. The yield of hydrated electrons at 30 picoseconds. Chem Lett. 1982;11:1887-90.

[30] LaVerne JA, Pimblott SM. Scavenger and time dependences of radicals and molecular products in the electron radiolysis of water: examination of experiments and models. J Phys Chem. 1991;95:3196-206.

[31] Belloni J, Billiau F, Delaire JA, Delcourt MO, Marignier JL. Ionizing radiation-liquid interactions: a comparative study of polar liquids. Radiat Phys Chem. 1983;21:177-83.

[32] Elliot AJ, Chenier MP, Ouellette DC. Temperature dependence of $\mathrm{g}$ values for $\mathrm{H} 2 \mathrm{O}$ and $\mathrm{D} 2 \mathrm{O}$ irradiated with low linear energy transfer radiation. J Chem Soc Faraday Trans. 1993;89:1193-7.

[33] Tomita H, Kai M, Kusama T, Ito A. Monte Carlo simulation of physicochemical processes of liquid water radiolysis. Radiat Environ Bioph. 1997;36:105-16.

[34] Pimblott SM, LaVerne JA. Stochastic simulation of the electron radiolysis of water and aqueous solutions. J Phys Chem A. 1997;101:5828-38.

[35] Bartels DM, Cook AR, Mudaliar M, Jonah CD. Spur decay of the solvated electron in picosecond radiolysis measured with time-correlated absorption spectroscopy. J Phys Chem A. 2000;104:1686-91.

[36] Jay-Gerin J-P, Ferradini C. A new estimate of the $\mathrm{OH}$ radical yield at early times in the radiolysis of liquid water. Chem Phys Lett. 2000;317:388-91.

[37] LaVerne JA. OH radicals and oxidizing products in the gamma radiolysis of water. Radiat Res. 2000;153:196200.

[38] Muroya Y, Lin M, Wu G, Iijima H, Yoshii K, Ueda T, et al. A re-evaluation of the initial yield of the hydrated electron in the picosecond time range. Radiat Phys Chem. 2005;72:169-72.

[39] Wang F, Schmidhammer U, Larbre JP, Zong Z, Marignier JL, Mostafavi M. Time-dependent yield of the hydrated electron and the hydroxyl radical in D2O: a picosecond pulse radiolysis study. Phys Chem Chem Phys. 2018.

[40] Schwarz HA, Caffrey Jr JM, Scholes G. Radiolysis of neutral water by cyclotron produced deuterons and helium ions. J American Chem Soc. 1959;81:1801-9.

[41] Naleway CA, Sauer Jr MC, Jonah CD, Schmidt KH. Theoretical analysis of the LET dependence of transient 
yields observed in pulse radiolysis with ion beams. Radiat Res. 1979;77:47-61.

[42] Burns WG, Sims HE. Effect of radiation type in water radiolysis. J Chem Soc Faraday Trans. 1981;77:2803-13. [43] Wasselin-Trupin V, Baldacchino G, Bouffard S, Hickel B. Hydrogen peroxide yields in water radiolysis by high-energy ion beams at constant LET. Radiat Phys Chem. 2002;65:53-61.

[44] Anderson AR, Hart EJ. Molecular product and free radical yields in the decomposition of water by protons, deuterons, and helium ions. Radiat Res. 1961;14:689-704.

[45] Pastina B, LaVerne JA. Hydrogen peroxide production in the radiolysis of water with heavy ions. J Phys Chem A. 1999;103:1592-7.

[46] Schwarz HA. Applications of the spur diffusion model to the radiation chemistry of aqueous solutions. J Phys Chem. 1969;73:1928-37.

[47] Sakata D, Belov O, Bordage MC, Emfietzoglou D, Guatelli S, Inaniwa T, et al. Fully integrated Monte Carlo simulation for evaluating radiation induced DNA damage and subsequent repair using Geant4-DNA. Sci Rep. 2020;10:20788.

[48] Montenegro EC, Scully SWJ, Wyer JA, Senthil V, Shah MB. Evaporation, fission and auto-dissociation of doubly charged water. J Electron Spectrosc. 2007;155:81-5.

[49] Meesungnoen J, Jay-Gerin JP. High-LET radiolysis of liquid water with $1 \mathrm{H}+, 4 \mathrm{He} 2+, 12 \mathrm{C} 6+$, and 20Ne9+ ions: effects of multiple ionization. J Phys Chem A. 2005;109:6406-19. 